\title{
Transformation optics for surface phenomena: Engineering the Goos-Hänchen effect
}

\author{
Lieve Lambrechts, ${ }^{1}$ Vincent Ginis, ${ }^{1}$ Jan Danckaert, ${ }^{1}$ and Philippe Tassin ${ }^{1,2, *}$ \\ ${ }^{1}$ Applied Physics Research Group, Vrije Universiteit Brussel (VUB), Pleinlaan 2, B-1050, Brussel, Belgium \\ ${ }^{2}$ Department of Physics, Chalmers University of Technology, SE-412 96, Göteborg, Sweden \\ (Received 20 October 2016; revised manuscript received 9 January 2017; published 26 January 2017)
}

\begin{abstract}
Transformation optics, a geometrical recipe for metamaterial design, was originally conceived as a tool to smoothly modify the trajectory of light using continuous coordinate transformations. Here, we show how discontinuous transformations can be used as a geometric framework to understand and manipulate phenomena at the surface of nanophotonic structures. In particular, we show how the Goos-Hänchen shift—a lateral shift exhibited by totally reflected beams_ can be tailored and we provide a classification and complete analytical description of this effect in existing complex media.
\end{abstract}

DOI: 10.1103/PhysRevB.95.035427

Transformation optics [1-3] has significantly advanced the field of metamaterials [4-7] by virtue of its elegant and intuitive design procedure based on geometrical deformations. After its conception in 2006, the development of several transformation-optical devices quickly followed, among which the invisibility cloak [8-12] probably appeals most to the imagination. These devices implement continuous coordinate transformations, allowing for reflectionless modification of light beam trajectories [13]. In addition, recent applications successfully manipulate a wide variety of electromagnetic effects [14-25] as well as effects in other paradigms of physics, hereby extending the scope of transformation optics far beyond its initial purpose. Indeed, the idea underlying transformation optics can be generalized to other fields of physics that are governed by equations which transform invariantly under coordinate transformations. Transformation physics in combination with recent advances in artificial materials [26] has revolutionized nanomechanics, heat flow physics, acoustics, and fluid dynamics [27-30]. Until now, transformation optics was mainly used to modify the trajectory of light inside three-dimensional media [1-3] and to manipulate the path of surface waves [31-35]. Transformation optics is deemed unable to deal with surfaces; surfaces were rather seen as a hindrance causing unwanted reflections. The use of transformation optics for the understanding and engineering of surface phenomena at the interface between complex media has remained unexplored.

In this article, we demonstrate that transformation optics can be used to engineer a surface phenomenon by using discontinuous coordinate transformations. Furthermore, we will show how the geometrical paradigm can be used to understand and describe this phenomenon at the interface of existing complex media. Our unconventional application of transformation optics will result in unprecedented understanding and control of the Goos-Hänchen effect-an interference phenomenon where a totally reflected beam is shifted from its predicted position. This effect was discovered experimentally by Hermann Goos and Hilda Hänchen in 1947 [36] and formalized theoretically by Kurt Artmann [37] the following year. Since then, the Goos-Hänchen effect has been studied in various optical media [38-45], including man-made

\footnotetext{
* Corresponding author.
}

materials such as left-handed media [46] and epsilon-near-zero metamaterials [47]. In the recent literature, the Goos-Hänchen effect is used for many practical purposes, underlying the design of optical devices that improve waveguiding [48-50], sensing [51], ground-plane cloaking [52], and holography [53].

First, we calculate the Goos-Hänchen shift for a transformation medium implementing a local linear transformation of Cartesian coordinates on a background of empty space. Subsequently, we investigate the roles of the constituting geometrical parameters and offer practicable alternatives in two special cases. Finally, we will use transformation optics to describe the Goos-Hänchen effect in biaxial $\overline{\bar{\varepsilon}} / \varepsilon_{0}=\overline{\bar{\mu}} / \mu_{0}$ media from a geometrical point of view.

In our geometric investigation of the Goos-Hänchen effect, we let an electromagnetic beam, incident from vacuum, reflect on a scaled transformation medium. To this end, we perform a local discontinuous scaling of the Cartesian coordinate lines on empty space:

$$
\begin{aligned}
& x^{\prime}=x, y^{\prime}=y, z^{\prime}=z \quad \text { for } x<0, \\
& x^{\prime}=a_{x} x, y^{\prime}=a_{y} y, z^{\prime}=a_{z} z \quad \text { for } x>0,
\end{aligned}
$$

where $a_{x}, a_{y}$, and $a_{z}$ are positive real numbers. The equivalence relations of transformation optics [1,2] then yield the relative permittivity and permeability tensors for the material implementation of the scaled half-space, which are inherently equal. Specifically, the aformentioned coordinate transformation can be implemented by a vacuum half-space for $x<0$ and a biaxial anisotropic medium with

$$
\begin{aligned}
& \varepsilon_{x x} / \varepsilon_{0}=\mu_{x x} / \mu_{0}=a_{y} a_{z} / a_{x}, \\
& \varepsilon_{y y} / \varepsilon_{0}=\mu_{y y} / \mu_{0}=a_{x} a_{z} / a_{y}, \\
& \varepsilon_{z z} / \varepsilon_{0}=\mu_{z z} / \mu_{0}=a_{x} a_{y} / a_{z},
\end{aligned}
$$

for $x>0$ as shown in Fig. 1.

Since traditional transformation optics in principle does not incorporate the effects of local discontinous transformations on the propagation of light [54], we rely on a first-principles approach applying Maxwell's boundary conditions at the interface between the empty half-space and the transformation medium. For a TE polarized plane wave, incident on the scaled transformation medium under an angle $\theta_{I}$ with the interface normal (see Fig. 1), we obtain the reflection coefficient

$$
r=\frac{2 \cos \theta_{I}}{\cos \theta_{I}+\frac{a_{y}}{a_{z}} \cos \left(\sin ^{-1}\left(\frac{1}{a_{y}} \sin \theta_{I}\right)\right)}-1,
$$




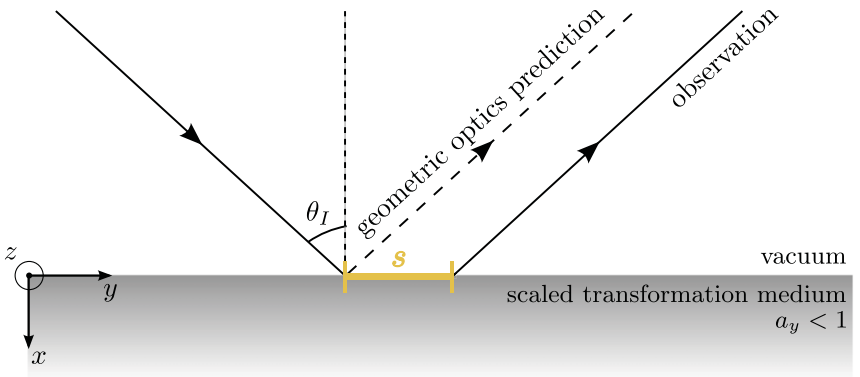

FIG. 1. Electromagnetic beam, incident upon an optically less dense medium, is shifted from its position predicted by geometric optics upon total reflection. Due to coherence effects, the finite beam is shifted over a distance $s$ along the interface, known as the GoosHänchen shift.

expressed solely as a function of the $y$ and $z$ scaling factors and incident angle. With the scaled transformation medium instrinsically having identical electric and magnetic responses, Eq. (3) remains valid for the TM mode.

If $a_{y}<1$, which corresponds to a stretching of the $y$ coordinate lines by $1 / a_{y}$, the reflection coefficient becomes 1 for incident angles larger than the critical angle $\sin ^{-1}\left(a_{y}\right)$ (see the Supplemental Material [55]). The total reflection regime is thus fully determined by the value of $a_{y}$, the scaling factor in the $y$ direction. Figure 2 presents the magnitude $|r|$ and phase $\phi_{r}$ of the reflection coefficient, supported by the results of full-wave numerical simulations in the total reflection regime. Moreover, if $a_{y}>a_{z}$, electromagnetic waves incident at Brewster's angle $\theta_{\mathrm{B}}=\cos ^{-1}\left(\sqrt{\left(a_{y}^{2}-1\right) /\left(a_{z}^{2}-1\right)}\right)$ are fully transmitted [55], as seen in Figs. 2(b) and 2(d).

The variation of the phase with respect to the incident angle in the total reflection regime $\left[\theta_{I}>\sin ^{-1}\left(a_{y}\right)\right]$ will cause the different spectral components of a finite incident beam to undergo different phase shifts, effectuating a shift of the

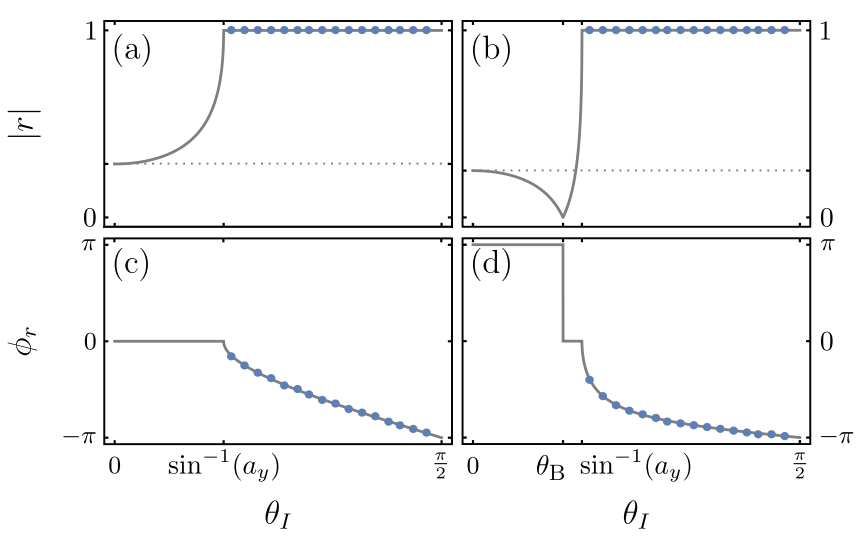

FIG. 2. Analytical predictions (gray curves) of the magnitude $|r|$ [(a), (b)] and the phase $\phi_{r}[(\mathrm{c}),(\mathrm{d})]$ of the reflection coefficient as a function of the angle of incidence $\theta_{I}$, supported by the results of fullwave numerical simulations (blue dots) in the total reflection regime. If $a_{y}>a_{z}[(\mathrm{~b}),(\mathrm{d})]$, electromagnetic waves incident at Brewster's angle $\theta_{\mathrm{B}}$ are fully transmitted. There is no such angle if $a_{y}<a_{z}$ $[(\mathrm{a}),(\mathrm{c})]$. The dotted lines in $[(\mathrm{a}),(\mathrm{b})]$ represent the respective values of $|r|$ at normal incidence, given by $\left|\left(a_{y}-a_{z}\right) /\left(a_{y}+a_{z}\right)\right|$.
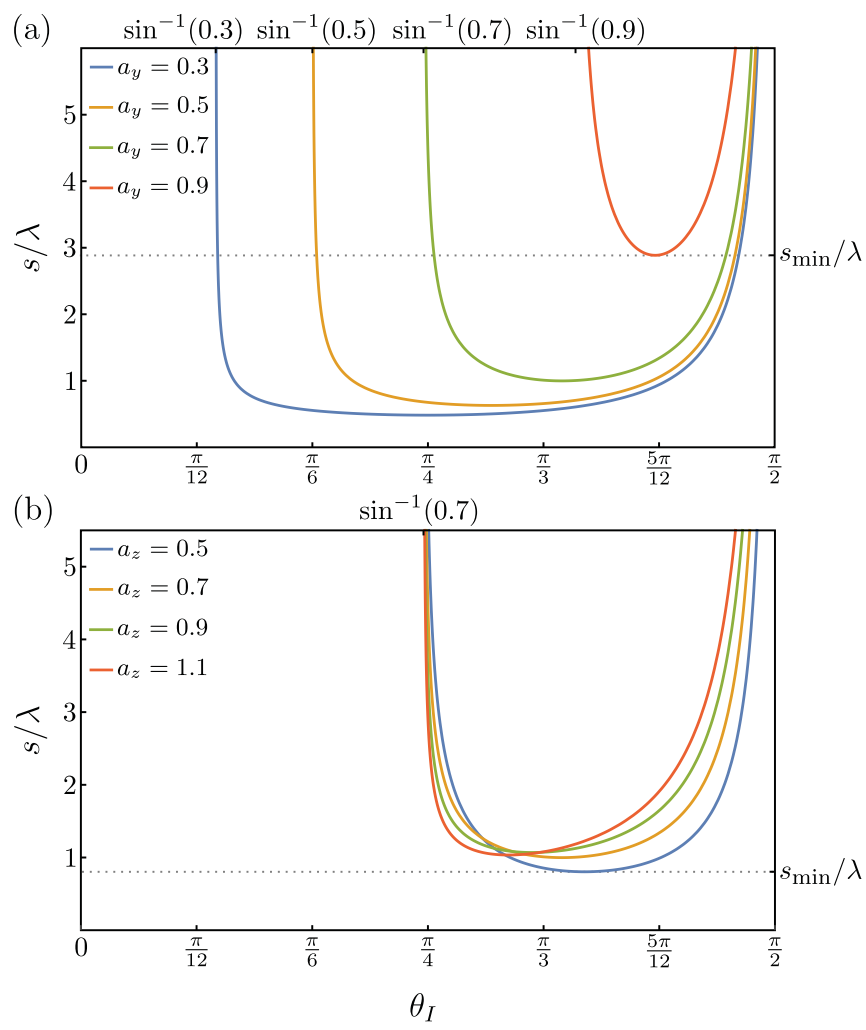

FIG. 3. Goos-Hänchen shift $s$, represented as a number of incident wavelengths $\lambda$, is plotted as a function of the angle of incidence $\theta_{I}$ for different values of the scaling factors. (a) Increasing values of $a_{y}$ show a significant increase of $s$, while the total reflection regime, determined by $\sin ^{-1}\left(a_{y}\right)$, decreases. The dotted line marks the value of the minimal Goos-Hänchen shift $s_{\min }$ for $a_{y}=0.9$ and $a_{z}=0.7$. (b) Varying $a_{z}$ slightly tunes $s$, while the total reflection regime remains unchanged. The critical angle is determined by the value of $a_{y}$, set here at 0.7 . The minimal Goos-Hänchen shift $s_{\min }$ for $a_{y}=0.7$ and $a_{z}=0.5$ is marked by the dotted line.

reflected beam along the interface: the Goos-Hänchen shift (see Fig. 1). The size $s$ of the shift can be derived from Eq. (3) using Artmann's formula [37]. We obtain [55]

$$
s=\frac{\lambda\left(a_{y}^{2}-1\right) a_{z} \tan \theta_{I}}{\pi a_{y}\left(a_{y}^{2}-a_{z}^{2} \cos ^{2} \theta_{I}-\sin ^{2} \theta_{I}\right) \sqrt{\frac{1}{a_{y}^{2}} \sin ^{2} \theta_{I}-1}},
$$

expressed as a function of the incident wavelength $\lambda$. Note that the scaling of the $x$ coordinates does not contribute to the Goos-Hänchen effect in agreement with our physical intuition.

The effect of the $y$ and $z$ scaling factors on the GoosHänchen shift $s$ is illustrated in Fig. 3. Increasing values of $a_{y}$ show a significant increase of $s$, whereas the regime in which the shift occurs - determined by the critical angle $\sin ^{-1}\left(a_{y}\right)$ diminishes [see Fig. 3(a)]. Varying $a_{z}$ induces minor changes of $s$, while the total reflection regime remains unchanged as can be seen in Fig. 3(b). This behavior is also reflected in Fig. 4, which depicts $s_{\min }\left(a_{y}, a_{z}\right)$, the minimal Goos-Hänchen shift that occurs for a given value of $a_{y}$ and $a_{z}$. Clearly, the leading role is played by $a_{y}$ : if $a_{y}$ approaches unity, the minimal shift increases dramatically. 

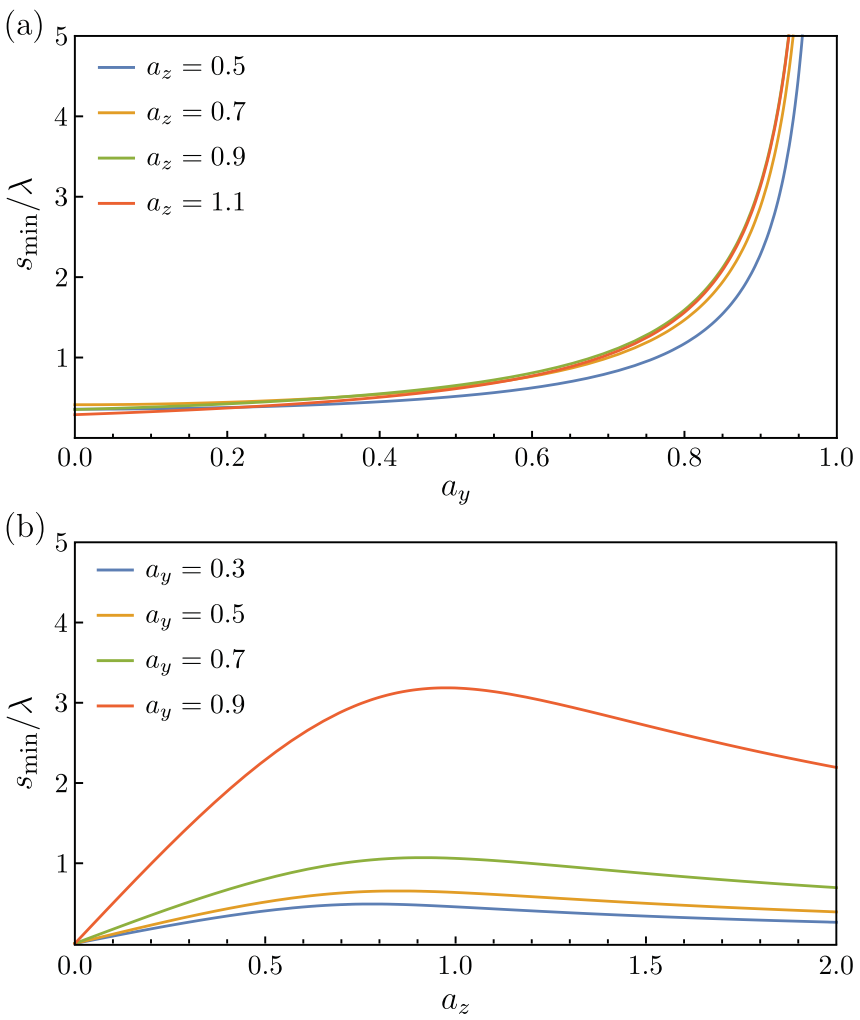

FIG. 4. Evolution of the minimal Goos-Hänchen shift $s_{\min }$. (a) When $a_{y}$ approaches unity, $s_{\min }$ dramatically increases. (b) Tuning $a_{z}$ allows for optimization of the minimal shift obtained for a certain $y$ scaling factor $a_{y}$.

To corroborate these results, we performed numerical simulations [55] of a beam with a Gauss-Hermite profile, incident on an isotropically stretched transformation medium $\left(a_{x}=a_{y}=a_{z}<1\right)$. Values for $s$ [blue dots in Fig. 5(a)] were obtained directly from the spatial distribution of the electric field norm [Fig. 5(b)].

Subsequently, we highlight two specific classes of scaled transformation media. First, if $a_{z}=1$ (i.e., the $z$-coordinate lines remain unaltered), Eq. (3) reduces to

$$
r=\frac{2 \cos \theta_{I}}{\cos \theta_{I}+a_{y} \cos \left(\sin ^{-1}\left(\frac{1}{a_{y}} \sin \theta_{I}\right)\right)}-1,
$$

the equation for the reflection coefficient $r_{\mathrm{TE}}$ for light incident from vacuum on a nonmagnetic medium with refractive index $n=a_{y}$. Hence the same phase profile and, therefore, also the same Goos-Hänchen shift profile, can be obtained from isotropic nonmagnetic media, which are considerably easier to manufacture. The correspondence of the phase profiles is demonstrated through full-wave numerical simulations, shown in Fig. 6(a). Medium A (blue circles) is a scaled transformation medium with $a_{x}=\sqrt{2.8}, a_{y}=\sqrt{0.7}$, and $a_{z}=1$. Medium $\mathrm{B}$ (blue triangles) is an isotropic nonmagnetic medium with refractive index $n=\sqrt{0.7}=a_{y}$. Both media yield an identical Goos-Hänchen shift profile (red curve).

Second, for $a_{z}=a_{y}^{2}$, Eq. (3) becomes

$$
r=\frac{2 \cos \theta_{I}}{\cos \theta_{I}+\frac{1}{a_{y}} \cos \left(\sin ^{-1}\left(\frac{1}{a_{y}} \sin \theta_{I}\right)\right)}-1,
$$

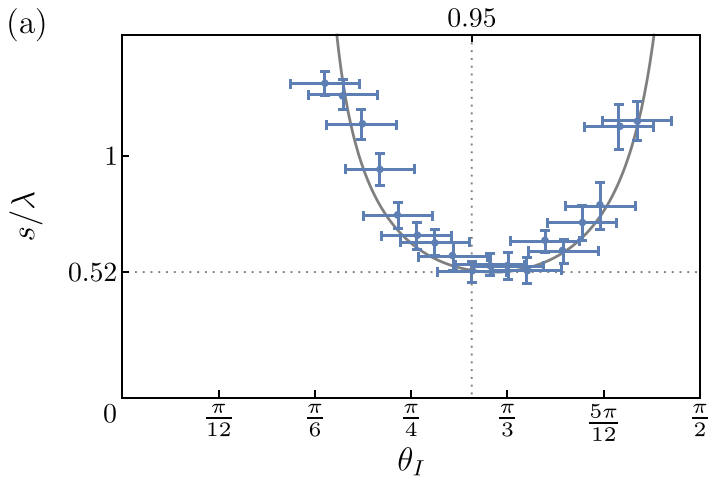

(b)

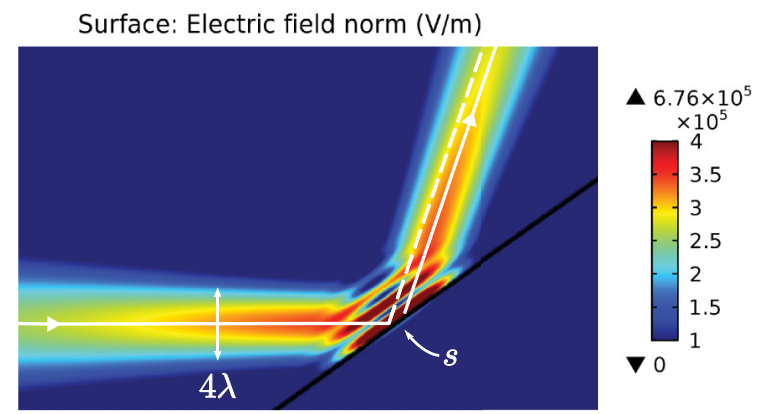

FIG. 5. (a) Goos-Hänchen shift $s$ is shown for reflection upon an isotropically stretched transformation medium $\left(a_{x}=a_{y}=a_{z}=0.5\right)$. The gray curve shows the analytical model, while the blue dots represent the results of finite-beam numerical simulations. (b) A GaussHermite beam is incident at $\theta_{I}=0.95 \mathrm{rad}$ on the stretched transformation medium, implemented by a magnetic medium with $\varepsilon / \varepsilon_{0}=$ $\mu / \mu_{0}=0.5$. The totally reflected beam is shifted by $s=0.52 \lambda$.

which corresponds to the reflection coefficient $r_{\mathrm{TM}}$ we would obtain for a nonmagnetic medium with refractive index $n=a_{y}$ for TM polarization. The agreement of the respective phase profiles is demonstrated in Fig. 6(b). Full-wave numerical simulations show coinciding phase profiles for TM reflection on medium $\mathrm{C}$ (blue circles) and medium $\mathrm{D}$ (blue triangles). The condition $a_{z}=a_{y}^{2}$ is satisfied by medium C: a stretched medium with $a_{x}=\sqrt{8.1}, a_{y}=\sqrt{0.9}$, and $a_{z}=0.9$. An identical Goos-Hänchen shift profile (red curve) is obtained by the isotropic nonmagnetic medium $\mathrm{D}$ with refractive index $n=\sqrt{0.9}=a_{y}$.

The previous results indicate how transformation optics can be used to design (meta)materials with a desired GoosHänchen effect from a geometrical point of view. In the language of transformation optics, the Goos-Hänchen shift is shown to depend only on two geometrical parameters, hereby significantly simplifying the design procedure. In the final part of this article, we will show the reverse procedure: using transformation optics to obtain a clear and concise analytical description of the Goos-Hänchen effect in existing materials by translating the material parameters into their geometrical counterparts.

In particular, general biaxial media, for which the relative permittivity and permeability tensors are equal, relate to the following scaling factors: $a_{x}=\sqrt{\varepsilon_{y y} \varepsilon_{z z}} / \varepsilon_{0}, a_{y}=\sqrt{\varepsilon_{x x} \varepsilon_{z z}} / \varepsilon_{0}$, and $a_{z}=\sqrt{\varepsilon_{x x} \varepsilon_{y y}} / \varepsilon_{0}$. The biaxial medium can thus be seen as a material implementation of a local coordinate transformation of empty space, given by Eq. (1). The aforementioned results 

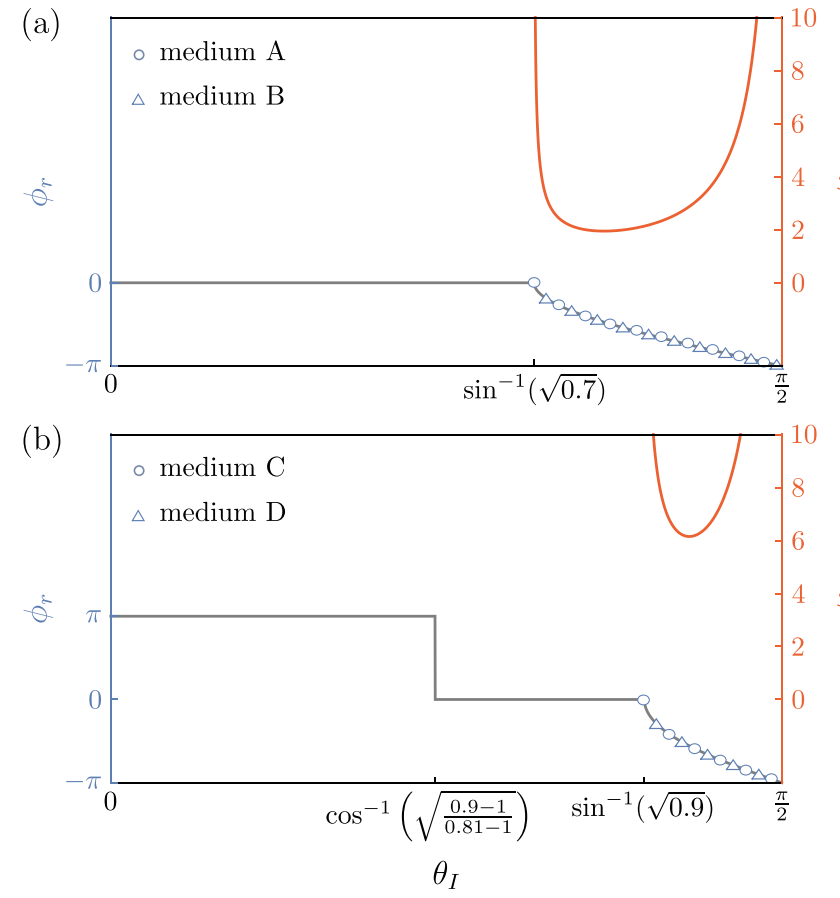

FIG. 6. For certain values of $a_{z}$, the reflection coefficient of the (anisotropic) scaled transformation medium matches that of an-easier to manufacture-isotropic nonmagnetic medium. The identical phase profile, verified by full-wave numerical simulations (blue markers), will give rise to identical Goos-Hänchen shifts (red curves). (a) A scaled transformation medium with $a_{z}=1$ (medium A) and an isotropic nonmagnetic medium with refractive index $n=a_{y}$ (medium B) generate identical Goos-Hänchen shifts for TE light. (b) A stretched transformation medium with $a_{z}=a_{y}^{2}$ (medium C) and an isotropic nonmagnetic medium with refractive index $n=a_{y}$ (medium D) have the same property for TM light.

then provide a complete description of the Goos-Hänchen effect of the biaxial medium, determined exclusively by $a_{y}$ and $a_{z}$.

Since $a_{x}$ is not a contributing factor, it provides a degree of freedom and hence allows for the classification of different biaxial media according to their associated GoosHänchen shift profile. As an example, we consider three different biaxial media: medium $\mathrm{E}\left(\varepsilon_{x x} / \varepsilon_{0}=2, \varepsilon_{y y} / \varepsilon_{0}=0.8\right.$, $\left.\varepsilon_{z z} / \varepsilon_{0}=0.2\right)$, medium $\mathrm{F}\left(\varepsilon_{x x} / \varepsilon_{0}=1, \varepsilon_{y y} / \varepsilon_{0}=1.6, \varepsilon_{z z} / \varepsilon_{0}=\right.$ $0.4)$, and medium $\mathrm{G}\left(\varepsilon_{x x} / \varepsilon_{0}=0.5, \varepsilon_{y y} / \varepsilon_{0}=3.2, \varepsilon_{z z} / \varepsilon_{0}=\right.$ $0.8)$. Due to matching associated scaling factors $a_{y}=\sqrt{0.4}$ and $a_{z}=\sqrt{1.6}$, these materials will exhibit identical GoosHänchen shifts, even though the associated $x$ scaling factors are different $\left(a_{x}=\sqrt{0.16}, \sqrt{0.64}\right.$, and $\sqrt{2.56}$, respectively). We solidify our claim by means of full-wave numerical simulations (see Fig. 7): medium E (blue circles), medium

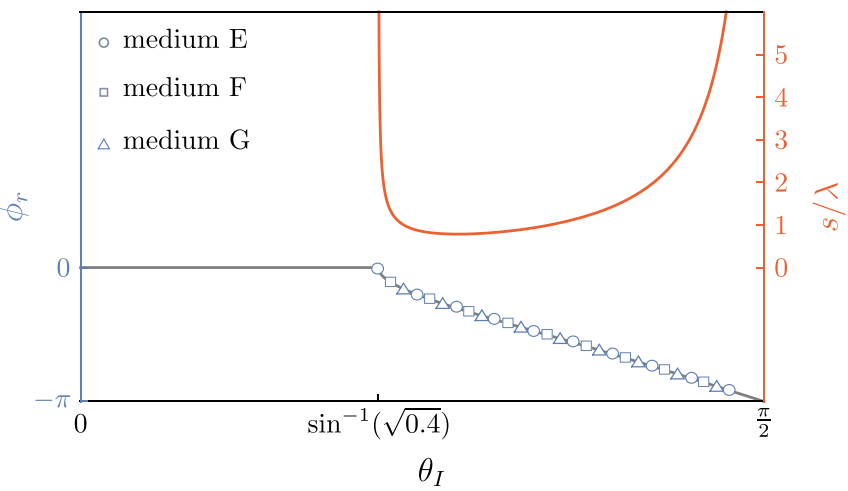

FIG. 7. Three different biaxial $\overline{\bar{\varepsilon}} / \varepsilon_{0}=\overline{\bar{\mu}} / \mu_{0}$ media relate to the same $y$ and $z$ scaling factors and thus yield identical GoosHänchen shifts. The matching phase profile was verified by full-wave numerical simulations for three different media: medium $\mathrm{E}\left(\varepsilon_{x x} / \varepsilon_{0}=\right.$ $\left.2, \varepsilon_{y y} / \varepsilon_{0}=0.8, \varepsilon_{z z} / \varepsilon_{0}=0.2\right), \mathrm{F}\left(\varepsilon_{x x} / \varepsilon_{0}=1, \varepsilon_{y y} / \varepsilon_{0}=1.6, \varepsilon_{z z} / \varepsilon_{0}=\right.$ $0.4)$, and $\mathrm{G}\left(\varepsilon_{x x} / \varepsilon_{0}=0.5, \varepsilon_{y y} / \varepsilon_{0}=3.2, \varepsilon_{z z} / \varepsilon_{0}=0.8\right)$. These media all yield the same $y$ and $z$ scaling factors: $a_{y}=\sqrt{0.4}$ and $a_{z}=\sqrt{1.6}$. The associated $x$ stretchings, however, are different. For medium E: $a_{x}=\sqrt{0.16}, \mathrm{~F}: a_{x}=\sqrt{0.64}$, and G: $a_{x}=\sqrt{2.56}$.

F (blue squares), and medium $\mathrm{G}$ (blue triangles) experience identical phase shifts and will thus yield the same GoosHänchen shifts (red curve).

In conclusion, we highlight two major advantages of applying transformation optics to the Goos-Hänchen effect. First, applying the transformation optics machinery on local discontinuous coordinate transformations opens up the road towards designer features at the interface of man-made materials. Second, the implicit investigation of complex media by means of their geometric counterparts provides a new perspective on existing phenomena, from which analytical models can readily be acquired. As an example, we demonstrated how the Goos-Hänchen effect can be designed by means of just two geometrical parameters, and, conversely, how these geometric parameters establish a full analytical description of the GoosHänchen effect at the interface of existing biaxial media. Contrary to common belief, we have shown that transformation optics is applicable at surfaces and not just inside bulk media. We envision that this use of the geometrical formalism can provide a new perspective on the design and understanding of electromagnetic surface phenomena [56-58].

V.G. acknowledges a fellowship from the Research Foundation Flanders (FWO-Vlaanderen). Work at VUB was also supported by the Interuniversity Attraction Poles program of the Belgian Science Policy Office under grant IAP P7-35 "photonics@be" and the research council (OZR) of the VUB.
[1] J. B. Pendry, D. Schurig, and D. R. Smith, Science 312, 1780 (2006).

[2] U. Leonhardt, Science 312, 1777 (2006).

[3] H. Chen, C. Chan, and P. Sheng, Nat. Mater. 9, 387 (2010).
[4] J. B. Pendry, A. J. Holden, D. J. Robbins, and W. J. Stewart, IEEE Trans. Microwave Theory Tech. 47, 2075 (1999).

[5] D. R. Smith, W. J. Padilla, D. C. Vier, S. C. Nemat-Nasser, and S. Schultz, Phys. Rev. Lett. 84, 4184 (2000). 
[6] M. Silveirinha and N. Engheta, Phys. Rev. Lett. 97, 157403 (2006).

[7] C. M. Soukoulis and M. Wegener, Nat. Photon. 5, 523 (2011).

[8] D. Schurig, J. J. Mock, B. J. Justice, S. A. Cummer, J. B. Pendry, A. F. Starr, and D. R. Smith, Science 314, 977 (2006).

[9] J. Li and J. B. Pendry, Phys. Rev. Lett. 101, 203901 (2008).

[10] H. Chen, B.-I. Wu, B. Zhang, and J. A. Kong, Phys. Rev. Lett. 99, 063903 (2007).

[11] H. Chen, B. Zheng, L. Shen, H. Wang, X. Zhang, N. I. Zheludev, and B. Zhang, Nat. Commun. 4, 2652 (2013).

[12] A. J. Danner, Opt. Express 18, 3332 (2010).

[13] M. Rahm, S. A. Cummer, D. Schurig, J. B. Pendry, and D. R. Smith, Phys. Rev. Lett. 100, 063903 (2008).

[14] D. A. Genov, S. Zhang, and X. Zhang, Nat. Phys. 5, 687 (2009).

[15] M. W. McCall, A. Favaro, P. Kinsler, and A. Boardman, J. Opt. 13, 024003 (2011).

[16] H. Chen and C. T. Chan, Phys. Rev. B 78, 054204 (2008).

[17] V. Ginis, P. Tassin, B. Craps, and I. Veretennicoff, Opt. Express 18, 5350 (2010).

[18] T. Zentgraf, Y. Liu, M. H. Mikkelsen, J. Valentine, and X. Zhang, Nat. Nanotechnol. 6, 151 (2011).

[19] P.-H. Tichit, S. N. Burokur, D. Germain, and A. de Lustrac, Phys. Rev. B 83, 155108 (2011).

[20] V. Ginis, P. Tassin, J. Danckaert, C. M. Soukoulis, and I. Veretennicoff, New J. Phys. 14, 033007 (2012).

[21] C. Sheng, H. Liu, Y. Wang, S. Zhu, and D. Genov, Nat. Photon. 7, 902 (2013).

[22] F. Liu, Z. Liang, and J. Li, Phys. Rev. Lett. 111, 033901 (2013).

[23] G. Castaldi, S. Savoia, V. Galdi, A. Alù, and N. Engheta, Phys. Rev. Lett. 110, 173901 (2013).

[24] V. Ginis, P. Tassin, C. M. Soukoulis, and I. Veretennicoff, Phys. Rev. Lett. 110, 057401 (2013).

[25] V. Ginis, J. Danckaert, I. Veretennicoff, and P. Tassin, Phys. Rev. Lett. 113, 167402 (2014).

[26] M. Kadic, T. Bückmann, R. Schittny, and M. Wegener, Rep. Prog. Phys. 76, 126501 (2013).

[27] H. Chen and C. Chan, Appl. Phys. Lett. 91, 183518 (2007).

[28] B.-I. Popa, L. Zigoneanu, and S. A. Cummer, Phys. Rev. Lett. 106, 253901 (2011).

[29] R. Schittny, M. Kadic, S. Guenneau, and M. Wegener, Phys. Rev. Lett. 110, 195901 (2013).

[30] H. Xu, X. Shi, F. Gao, H. Sun, and B. Zhang, Phys. Rev. Lett. 112, 054301 (2014).

[31] Y. Liu, T. Zentgraf, G. Bartal, and X. Zhang, Nano Lett. 10, 1991 (2010).

[32] A. Vakil and N. Engheta, Science 332, 1291 (2011).
[33] P. A. Huidobro, M. L. Nesterov, L. Martin-Moreno, and F. J. Garcia-Vidal, Nano Lett. 10, 1985 (2010).

[34] S. Xu, H. Xu, H. Gao, Y. Jiang, F. Yu, J. D. Joannopoulos, M. Soljačić, H. Chen, H. Sun, and B. Zhang, Proc. Natl. Acad. Sci. USA 112, 7635 (2015).

[35] S. Viaene, V. Ginis, J. Danckaert, and P. Tassin, Phys. Rev. B 93, 085429 (2016).

[36] F. Goos and H. Hänchen, Ann. Phys. (NY) 436, 333 (1947).

[37] K. Artmann, Ann. Phys. (NY) 437, 87 (1948).

[38] W. J. Wild and C. L. Giles, Phys. Rev. A 25, 2099 (1982).

[39] H. Lai and S. Chan, Opt. Lett. 27, 680 (2002).

[40] D. Felbacq, A. Moreau, and R. Smaâli, Opt. Lett. 28, 1633 (2003).

[41] L.-G. Wang, H. Chen, and S.-Y. Zhu, Opt. Lett. 30, 2936 (2005).

[42] J. He, J. Yi, and S. He, Opt. Express 14, 3024 (2006).

[43] M. Merano, A. Aiello, M. Van Exter, E. Eliel, J. Woerdman et al., Opt. Express 15, 15928 (2007).

[44] P. Leung, C. Chen, and H.-P. Chiang, Opt. Commun. 276, 206 (2007).

[45] K. Y. Bliokh and A. Aiello, J. Opt. 15, 014001 (2013).

[46] I. V. Shadrivov, A. A. Zharov, and Y. S. Kivshar, Appl. Phys. Lett. 83, 2713 (2003).

[47] Y. Xu, C. T. Chan, and H. Chen, Sci. Rep. 5, 8681 (2015).

[48] P. Tassin, X. Sahyoun, and I. Veretennicoff, Appl. Phys. Lett. 92, 203111 (2008).

[49] P. Wei, S. Xiao, Y. Xu, H. Chen, S. T. Chu, and J. Li, J. Opt. 18, 044015 (2016).

[50] S. J. Byrnes, M. Khorasaninejad, and F. Capasso, Opt. Express 24, 18399 (2016).

[51] S. A. Taya, E. J. El-Farram, and T. M. El-Agez, AEU-Int. J. Electron. Commun. 66, 204 (2012).

[52] B. Zhang, T. Chan, and B.-I. Wu, Phys. Rev. Lett. 104, 233903 (2010).

[53] C. Barsi, W. Wan, and J. W. Fleischer, Nat. Photon. 3, 211 (2009).

[54] L. Bergamin, Phys. Rev. A 80, 063835 (2009).

[55] See Supplemental Material at http://link.aps.org/supplemental/ 10.1103/PhysRevB.95.035427 for the analytical derivations of the critical angle, Brewster's angle and the Goos-Hänchen shift, and for the details of the finite elements numerical simulation of the Gauss-Hermite beam.

[56] A. Aiello, New J. Phys. 14, 013058 (2012).

[57] M. Merano, N. Hermosa, J. P. Woerdman, and A. Aiello, Phys. Rev. A 82, 023817 (2010).

[58] C. Menzel, C. Rockstuhl, T. Paul, S. Fahr, and F. Lederer, Phys. Rev. A 77, 013810 (2008). 